\title{
Ultrasound findings of noninvasive follicular thyroid neoplasm with papillary-like nuclear features compared with those of follicular variant of papillary carcinoma and encapsulated papillary carcinoma: a single-institution study in Japan
}

Maki Oshita ${ }^{1)}$, Yasuhiro Ito $^{2)}$, Tomoko Miyamoto ${ }^{1)}$, Hisashi Ota ${ }^{1)}$, Tomohiko Nakamura ${ }^{3)}$, Mitsuyoshi Hirokawa $^{4)}$, Akihiro Miya ${ }^{2)}$ and Akira Miyauchi ${ }^{2)}$

\author{
1) Departments of Clinical Laboratory, Kuma Hospital, Kobe 650-0011, Japan \\ 2) Departments of Surgery, Kuma Hospital, Kobe 650-0011, Japan \\ 3) Departments of Internal Medicine, Kuma Hospital, Kobe 650-0011, Japan \\ 4) Departments of Diagnostic Pathology, Kuma Hospital, Kobe 650-0011, Japan
}

\begin{abstract}
Noninvasive follicular thyroid neoplasm with papillary-like nuclear features (NIFTP) is a new entity adopted by the newest World Health Organization classification. It is differentiated from follicular variant papillary thyroid carcinoma (FVPTC) and regarded as non-malignant disease. Here, we compared the ultrasound findings of NIFTP $(n=40)$ with those of FVPTC $(n=94)$ and encapsulated PTC (encap-PTC) $(n=157)$. The NIFTP group showed benign findings on ultrasound significantly more frequently than the FVPTC group based on the Japan Society of Ultrasonics in Medicine criteria: a regular shape $(p<0.001)$, well edge definition $(p=0.007)$, smooth character $(p<0.001)$, isoechoic and homogeneous internal echoes $(p<0.001)$, lack of punctate microcalcification $(p=0.027)$, and a regular marginal hypoechoic zone $(p<0.001)$. Compared to encap-PTC, NIFTP has a significantly higher incidence of benign findings: isoechoic and homogeneous internal echoes $(p<$ $0.001)$, lack of punctate microcalcification $(p<0.001)$, and a regular marginal hypoechoic zone $(p=0.004)$. Based on the ultrasound classification (USC) system at Kuma Hospital, no cases were classified as malignant (USC $\geq 3.5$ ), but $55.4 \%$ of the FVPTCs and $53.5 \%$ of the encap-PTCs were diagnosed as malignancy. However, on cytology, the incidence of NIFTP classified as Bethesda-V or -VI (PTC) was very high at $86.9 \%$. All patients underwent surgical treatment, but none of the NIFTP patients showed postoperative recurrence. Although avoiding surgery might be difficult because of the high incidence of malignant cytology, overtreatment (including extensive surgery) for NIFTP can be avoided by paying close attention to the lack of malignant findings on ultrasound.
\end{abstract}

Key words: Noninvasive follicular thyroid neoplasm with papillary-like nuclear features (NIFTP), Encapsulated papillary Thyroid carcinoma, Follicular variant, Ultrasound

ON ULTRASOUND, papillary thyroid carcinoma (PTC) has some typical findings: irregular shape, illdefined margin, and microcalcifications. However, some subtypes such as follicular variant PTC (FVPTC), macrofollicular variant, and cribriform morular variant often lack such malignant findings on ultrasound, although most of these are suspected of being or diagnosed as PTC on cytology. Of these, FVPTC (which is

Submitted May 2, 2020; Accepted Jun. 19, 2020 as EJ20-0254 Released online in J-STAGE as advance publication Jul. 16, 2020 Correspondence to: Yasuhiro Ito, Department of Surgery, Kuma Hospital, 8-2-35 Shimoyamate-Dori, Chuo-ku, Kobe, Hyogo 650-0011, Japan.

E-mail: ito01@kuma-h.or.jp encapsulated and shows no invasive findings on pathological examination) was excluded from PTC category and regarded as benign after being renamed "noninvasive follicular thyroid neoplasm with papillary-like nuclear features (NIFTP) [1]. The diagnostic criteria of NIFTP are (1) encapsulation or clear demarcation, (2) follicular growth pattern with $<1 \%$ papillae, no psammoma bodies, and $<30 \%$ solid/trabecular/insular growth pattern, (3) nuclear score 2-3, (4) no vascular or capsular invasion, (5) no tumor necrosis, and (6) no high mitotic activity. NIFTP was recognized as a novel entity in the newest edition of the World Health Organization (WHO) classification [2]. The reported incidence of NIFTP in Western countries is $13.6 \%-25 \%$ among the cases previously 
diagnosed as PTC, which is much higher than the incidence in Asian countries at $0.8 \%$; the reasons for this disparity remain unclear [3].

Several studies have compared NIFTP and FVPTC (non-NIFTP) from various aspects including ultrasonic and pathological findings [4-16]. On ultrasound, the rate of findings suspected of malignancy in NIFTP was reported to be lower than that in non-NIFTP $[10,11$, 13-15]. In contrast, Hirokawa et al. showed that on cytology, $75.7 \%$ of NIFTP cases were reported as suspicious for malignancy or malignant [4]. We conducted the present study to investigate the clinical findings of NIFTP by focusing on the diagnostic criteria of ultrasound findings provided by the Japan Society of Ultrasonics in Medicine (JSUM) [17] and on the ultrasound classification (USC) system established at our institution, Kuma Hospital [18].

\section{Patients and Methods}

Between Jan. 2007 and Dec. 2016, 236 patients underwent surgery at Kuma Hospital and were pathologically diagnosed as having FVPTC. One of our coauthors (M.H.) reviewed their pathological specimens, and 58 of the cases were newly diagnosed as NIFTP. We enrolled 40 cases in this study after excluding the 14 cases with lesions measuring $<10 \mathrm{~mm}$ and another four cases with strong calcification (unsuitable for ultrasound evaluation). Of the remaining 178 cases with FVPTC (nonNIFTP), we excluded 54 cases with lesions $<10 \mathrm{~mm}$ and 30 cases with strong calcification, and we enrolled the remaining 94 cases for further analysis.

During the same period, 224 patients underwent surgery and were diagnosed as having encapsulated PTC (encap-PTC). We excluded 17 cases with lesions $<10 \mathrm{~mm}$, 34 cases with strong calcification, and 16 cases that were occupied mostly by a cystic lesion; we used the remaining 157 cases for further analyses. For the ultrasound examinations, we used an Aplio 80 SSA-770A or Aplio 500 TUS-A500 (Canon Medical Systems, Tochigi, Japan) as ultrasound machines and PLT-0805AT (5-12 MHz) and PLT-1005BT (5-14 MHz) (Canon Medical Systems) as linear-type probes, and an EUB-7500 (Hitachi, Tokyo) as an ultrasound machine with EUP-L65 (6$14 \mathrm{MHz}$ ) and EUP-L74M (5-13 MHz) (Hitachi) as linear-type probes.

For this study, one coauthor (M.O.) reviewed the ultrasound findings of all cases blindly. We investigated the nodules according to the JSUM diagnostic criteria and evaluated their shapes, boundary zones, internal echoes, fine strong echoes, and marginal hypoechoic zones [14]. We also examined Doppler signals for 39 NIFTP, 93 FVTC, and 157 encap-PTC patients.
The evaluation of elastography was subject to the classification provided by Asteria et al. [18]. We classified the nodules into four elastography scores (1 to 4). We set the cutoff between score 2 and 3 in order to divide the tumors into benign and malignant nodules.

We also used the ultrasound classification (USC) system established in 1996 at Kuma Hospital [19]. Briefly, nodules were classified into the following five categories. USC1: round and anechoic area (cyst or adenomatous nodule), USC2: round and cystic nodule (single or multiple) or isoechoic solid nodule (adenomatous nodule, or follicular adenoma), USC3: round and hypoechoic solid nodule (adenomatous nodule, follicular adenoma, or well-differentiated carcinoma), USC4: solid nodule with an irregular border or presence of psammoma calcification (carcinoma), and USC5: solid nodule with an irregular border and extrathyroidal invasion (carcinoma). Between USC2 and USC5, for nodules with ultrasound findings corresponding to two classes, we set intermediate classes, i.e., USC2.5, USC3.5, and USC4.5. Nodules in categories USC $\geq 3.5$ were diagnosed as malignant, those in category USC3 were diagnosed as borderline, and those in categories USC $\leq 2.5$ were diagnosed as benign.

Cytological findings were evaluated based on the Bethesda category [20]. We also investigated our surgery records and pathological reports for evaluations from the surgical and pathological aspects.

StatFlex software (View Flex, Tokyo) was used for all statistical analyses. The Mann-Whitney test and chisquare test were used to compare variables. Probability $(p)$ values $<0.05$ were considered significant.

\section{Results}

\section{Ultrasound findings}

The rate of male patients in the NIFTP group was $37.5 \%$ (15 of 40 patients), which was significantly higher than that in the FVPTC group (18.1\%, 17 of 94 patients; $p=0.016)$, but similar to that in the encap-PTC group $(32.5 \%, 51$ of 157 patients; $p=0.549)$. The median age of the NIFTP patients was 59 years (25-79), which was significantly older than that of the encap-PTC patients (median 47 years, 23-84 yrs; $p=0.002$ ) but similar to that of the FVPTC patients (median 60 years, 15-81 yrs; $p=0.703)$. The maximal diameter of the NIFTPs was similar to those of the FVPTCs $(p=0.054)$ and encapPTCs $(p=0.659)$.

Fig. 1 illustrates the groups' differences in ultrasound findings based on the JSUM diagnostic criteria. Compared to the FVPTC cases, the NIFTP cases more frequently showed benign findings on ultrasound: a regular shape $(p<0.001)$, well edge definition $(p=0.007)$, 


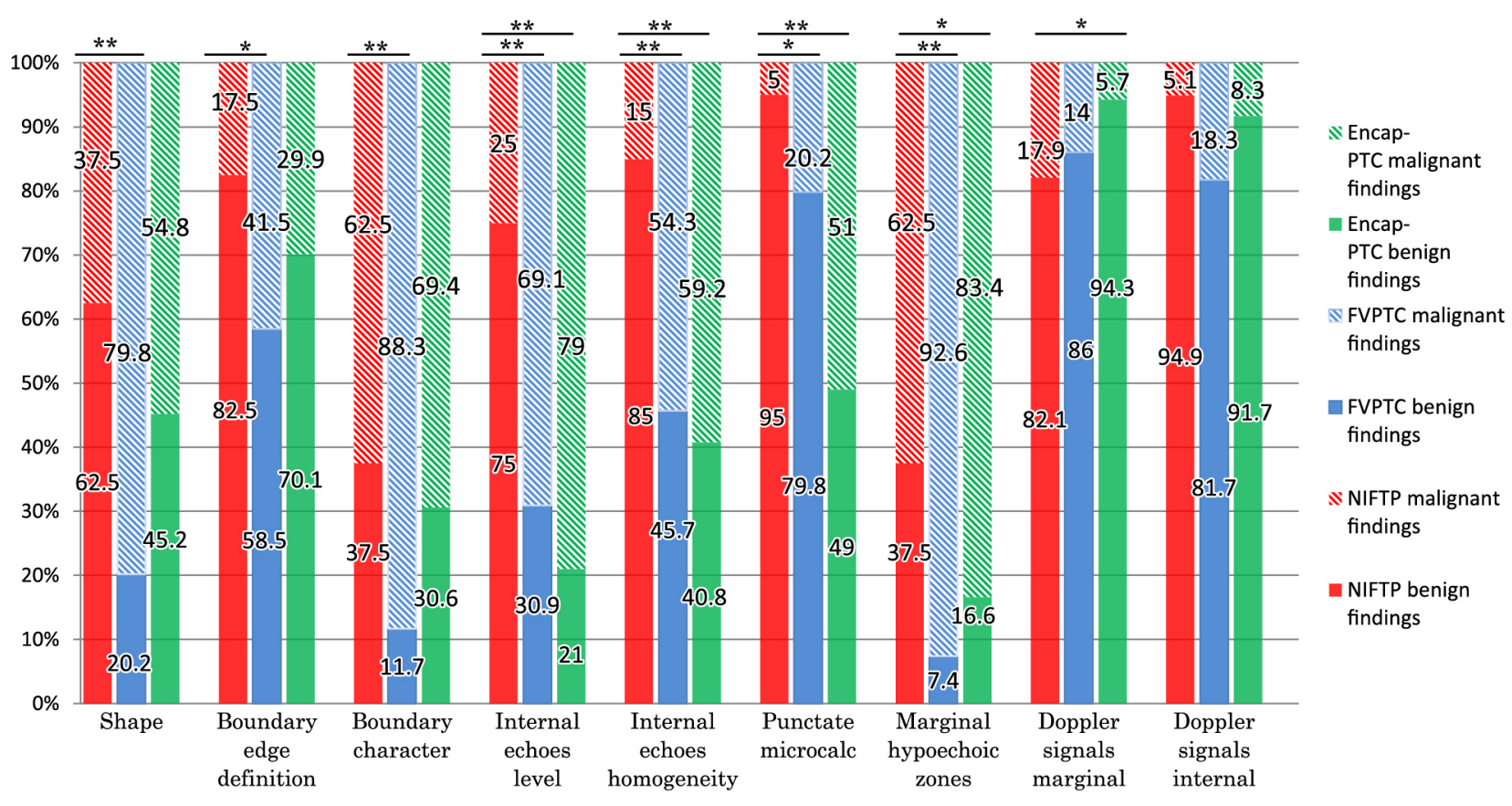

${ }^{\star} p<0.05,{ }^{* *} p<0.001$ (exact $p$-values are provided in the text).

Fig. 1 Comparison of ultrasound findings based on the JSUM diagnostic criteria for NIFTP with those for FVPTC and encap-PTC.

Table 1 Comparison of the number of benign ultrasound findings based on the JSUM criteria in the NIFTP group with those of the FVPTC and encap-PTC groups

\begin{tabular}{lccc|cc|ccc}
\hline No. of benign findings & 7 & 6 & 5 & 4 & 3 & 2 & 1 & 0 \\
\hline NIFTP, $n=40$ & $5(12.5 \%)$ & $9(22.5 \%)$ & $9(22.5 \%)$ & $8(20 \%)$ & $7(17.5 \%)$ & $1(2.5 \%)$ & $1(2.5 \%)$ & $0(0 \%)$ \\
FVPTC, $n=94$ & $1(1.1 \%)$ & $2(2.1 \%)$ & $9(9.6 \%)$ & $11(11.7 \%)$ & $20(21.3 \%)$ & $26(27.7 \%)$ & $17(18.1 \%)$ & $8(8.5 \%)$ \\
Encap-PTC, $n=157$ & $6(3.8 \%)$ & $10(6.0 \%)$ & $15(9.6 \%)$ & $23(14.6 \%)$ & $23(14.6 \%)$ & $30(19.1 \%)$ & $30(19.1 \%)$ & $20(12.7 \%)$ \\
\hline
\end{tabular}

The ultrasound criteria are seven ultrasound findings; shape, boundary (edge definition and character), Internal echoes (level and homogeneity), punctate microcalcification, and marginal hypoechoic zones. $p<0.001$ (NIFTP vs. FVPTC, NIFTP vs. encap-PC; cutoff points were set at 5 and 2).

smooth character $(p<0.001)$, isoechoic and homogeneous internal echoes $(p<0.001)$, lack of punctate microcalcification $(p=0.027)$, and a regular marginal hypoechoic zone $(p<0.001)$. Compared to the encapPTC cases, the NIFTP cases had a higher incidence of benign findings: isoechoic and homogeneous internal echoes $(p<0.001)$, lack of punctate microcalcification $(p<0.001)$, a regular marginal hypoechoic zone $(p=$ 0.004 ), although the incidence of lack of marginal Doppler signal of the NIFTP was even lower than that of the encap-PTC $(p=0.013)$.

Table 1 summarizes the results of our comparison of the number of benign ultrasound findings based on the JSUM criteria for NIFTP with those for FVPTC and encap-PTC. We set the cutoff points of the number of benign findings at 5 and 2 . The detection rate of NIFTP with $\geq 5$ benign findings was $57.5 \%(22.5 \%+22.5 \%$ $+12.5 \%$ ), which was significantly higher than those of
FVPTC and encap-PTC at $12.8 \%(9.6 \%+2.1 \%+1.1 \%)$ and $20.4 \%(9.6 \%+7.0 \%+3.8 \%)$, respectively $(p<$ 0.001 each). The detection rate of NIFTP with $\leq 2$ benign findings was $5.0 \%(0 \%+2.5 \%+2.5 \%)$, which was significantly lower than those of FVPTC and encap-PTC at $54.3(8.5 \%+18.1 \%+27.7 \%)$ and $50.3 \%(12.7 \%$ $+18.5 \%+19.1 \%)$, respectively $(p<0.001$ each $)$.

Fig. 2 provides the receiver operating characteristic (ROC) curve for the comparison of the number of benign findings according to the JSUM criteria for the NIFTP versus FVPTC groups (Fig. 2a) and the NIFTP versus encap-PTC groups (Fig. 2b). We calculated cutoff values for which the sensitivity equals the specificity: 3.06 (sensitivity and specificity $=0.763$ ) for NIFTP and FVPTC, and 3.34 (sensitivity and specificity $=0.706$ ) for NIFTP and encap-PTC.

Elastography was performed for some of the tumors: nine NIFTPs, nine FVPTCs, and 11 encap-PTCs. The 
(a)

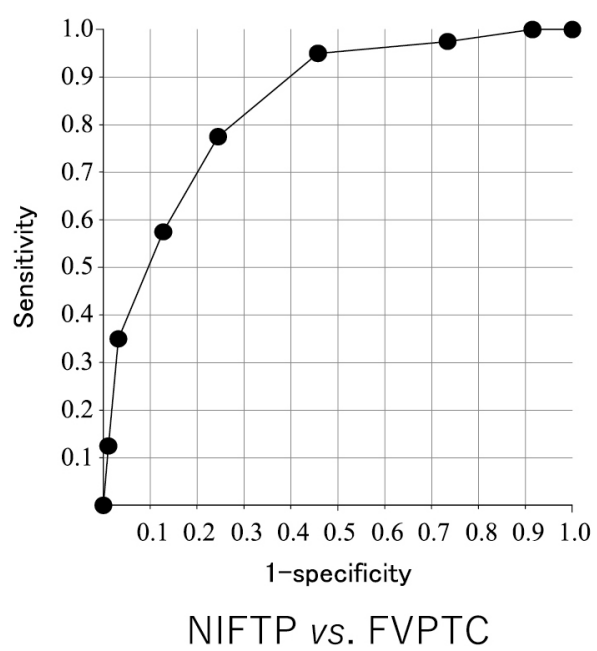

(b)

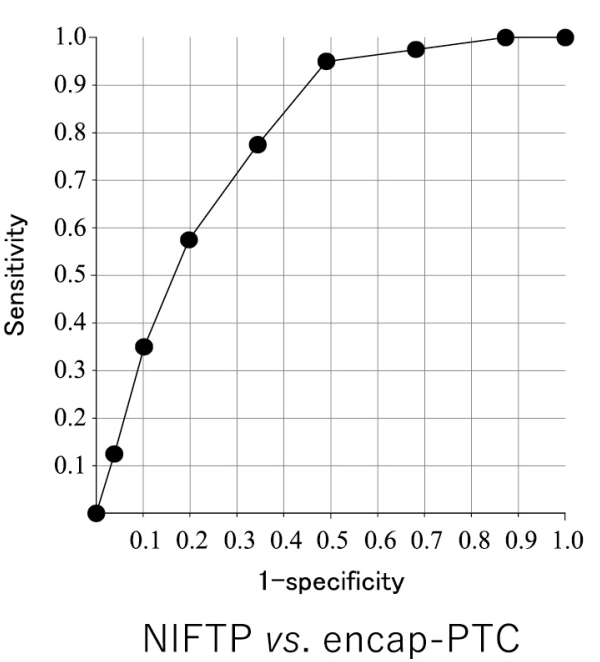

Fig. 2 ROC curves for the comparison of the number of benign ultrasound findings conducted by JSUM in the NIFTP group with those of the (a) FVPTC and (b) encap-PTC groups.

Table 2 Comparison of elastography findings for the NIFTP group with those of the FVPTC and encap-PTC groups

\begin{tabular}{lcc|cc}
\hline & \multicolumn{4}{c}{ NIFTP $v s$. FVPTC, $p=0.317$} \\
\cline { 2 - 5 } NIFTP $v s$. encap-PTC, $p=0.008$ \\
\hline Elastography score & 1 & 2 & \multicolumn{2}{c}{ Malignant } \\
NIFTP, $n=9$ & $2(22.2 \%)$ & $5(55.6 \%)$ & $1(11.1 \%)$ & $1(11.1 \%)$ \\
FVTPC, $n=9$ & $1(11.1 \%)$ & $4(44.4 \%)$ & $2(22.2 \%)$ & $2(22.2 \%)$ \\
Encap-PTC, $n=11$ & 0 & $2(18.2 \%)$ & $6(54.5 \%)$ & $3(27.3 \%)$ \\
\hline
\end{tabular}

Table 3 Comparison of Kuma Hospital US classification scores for the NIFTP group with those of the FVPTC and encap-PTC groups

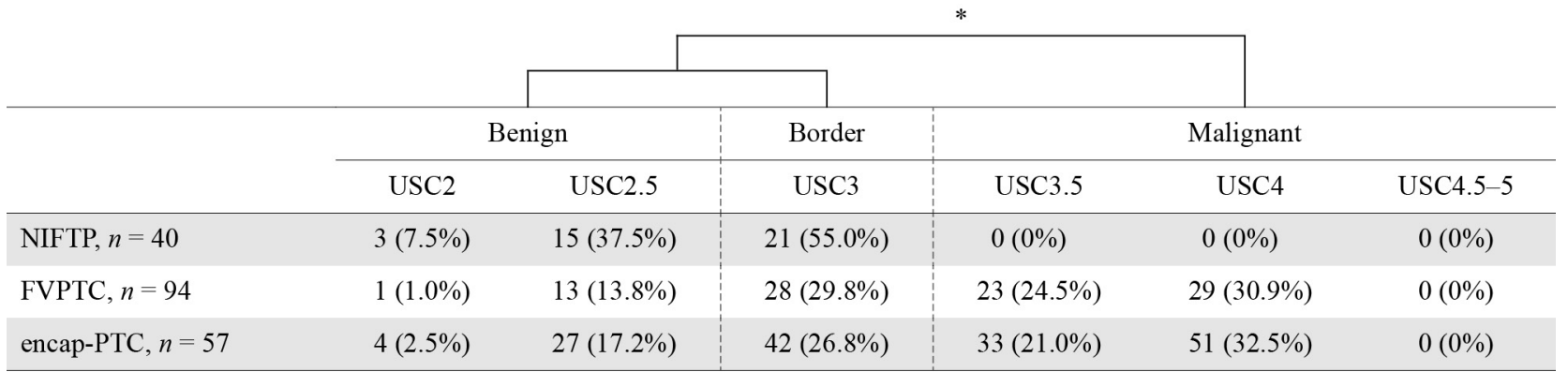

* NIFTP vs. FVPTC, $p<0.001$. NIFTP vs. encap-PTC, $p<0.001$.

elastography scores of the NIFTP did not differ significantly from those of the FVPTCs but were significantly lower $(p=0.008)$ than those of the encap-PTCs (Table 2).

Table 3 shows the results of the comparison of the USC categories of NIFTP with those of FVPTC and encap-PTC. No cases of NIFTP were classified as malignant (USC $\geq 3.5$ ) in our USC classification system, whereas $55.4 \%(24.5 \%+30.9 \%)$ of the FVPTC cases and $53.5 \%(21.0 \%+32.5 \%)$ of the encap-PTC cases were diagnosed as malignant. There were significant differences in the rates of benign and borderline cases on ultrasound between the NIFTP and FVPTC cases $(p<$ $0.001)$ and between the NIFTP and encap-PTC cases ( $p$ $<0.001)$. 

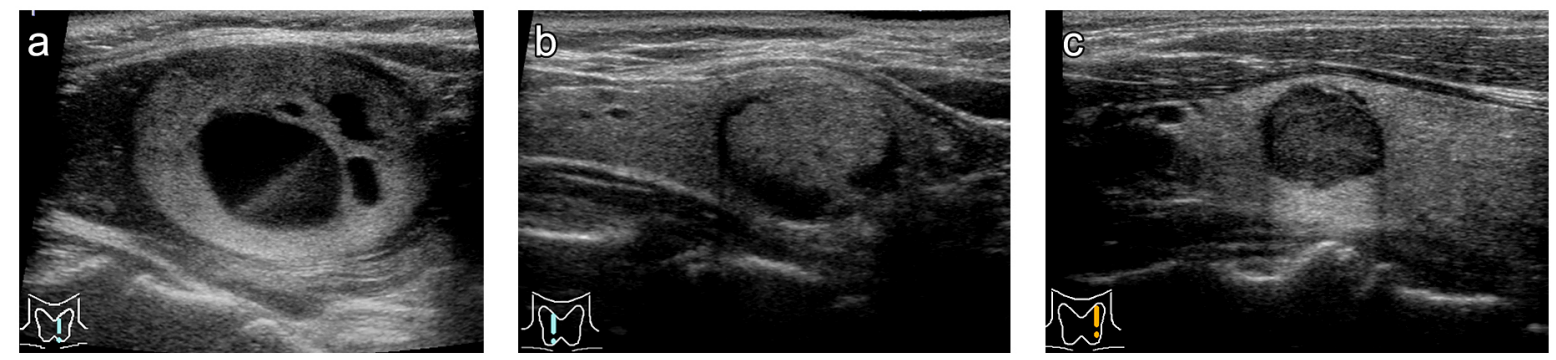

Fig. 3 a: Ultrasound imaging of an NIFTP. This has all seven benign findings based on the JSUM criteria and was classified as USC2. b: Ultrasound imaging of NIFTP. This nodule has two malignant findings based on the JSUM criteria (jagged boundary character and slightly irregular marginal hypoechoic zones) and was classified as USC3. c: Ultrasound imaging of NIFTP. This nodule has three malignant findings based on the JSUM criteria (slightly irregular shape, jagged boundary character and hypoechoic internal echoes) and was classified as USC3.
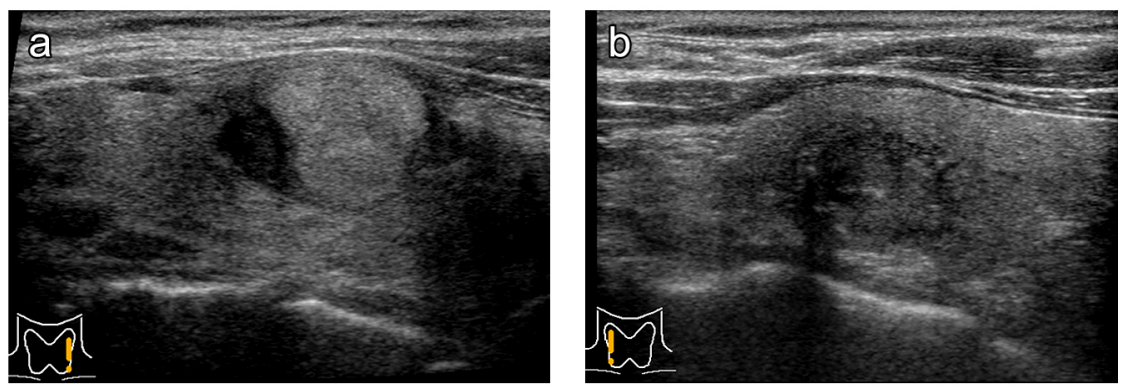

Fig. 4 a: Ultrasound imaging of an FVPTC with three malignant findings (irregular shape, partially hypoechoic, and heterogeneous internal echoes) which was classified as USC3. b: Ultrasound imaging of an FVPTC with all seven malignant findings which was classified as USC3.5.
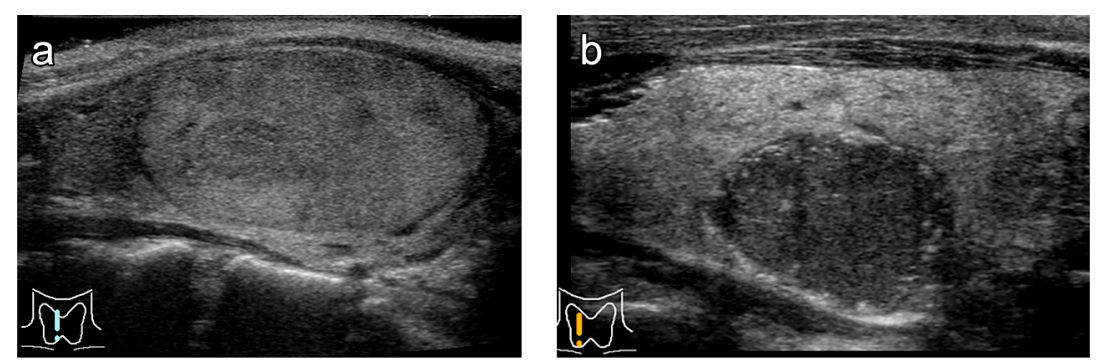

Fig. 5 a: Ultrasound imaging of an encap-PTC with no malignant findings which was classified as USC3. b: Ultrasound imaging of an encap-PTC with five malignant findings (the exceptions were good edge definition and homogenous internal echoes). This nodule was classified as USC3.5.

Figs. 3-5 are ultrasound images of representative cases of NIFPT, FVPTC, and encap-PTC, respectively.

\section{Cytological findings}

Table 4 provides the cytological findings of the patients with NIFTP $(n=38)$, FVPTC $(n=93)$, and encap-PTC $(n=156)$ who underwent fine-needle aspiration cytology. Of the 38 NIFTP patients, 28 (73.7\%) and five $(13.2 \%)$ patients were classified as Bethesda $(\mathrm{Be})$ VI (PTC) and Be-V, respectively. The detection rate of malignant cytology of NIFTP was high at $86.9 \%$ (13.2\%
$+73.7 \%$ ), which was similar to the finding for FVPTC $(p=0.3283)$, and even lower $(p=0.001)$ than that for encap-PTC.

\section{Surgery for NIFTP patients}

Of the 40 patients with NIFTP, $32(80.0 \%)$ had no coexisting carcinoma lesions in the thyroid on preoperative imaging studies. A total thyroidectomy was performed for 11 patients, and the remaining 21 underwent a lobectomy with or without isthmectomy. Twenty-nine patients underwent prophylactic central node dissection. 
Table 4 Relationships among the cytology findings of the NIFTP, FVPTC, and encap-PTC groups based on the Bethesda classification

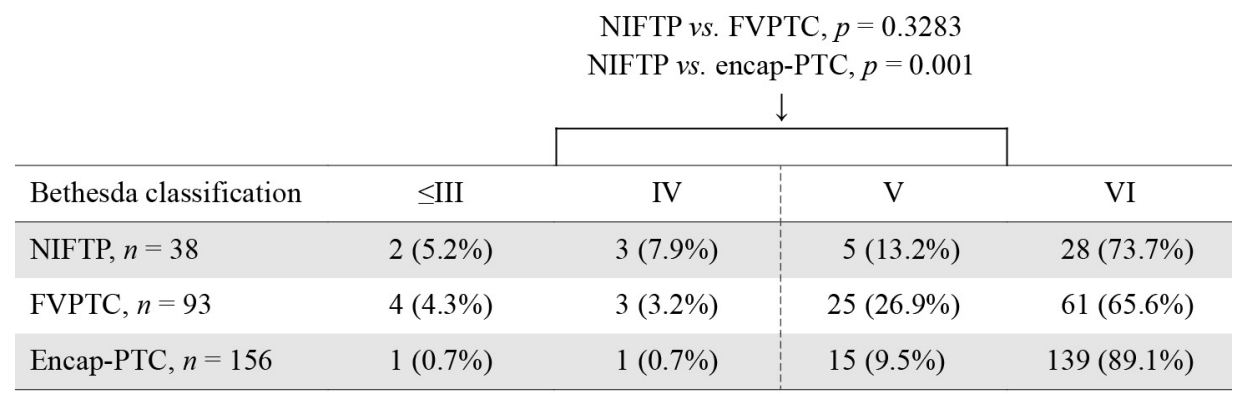

Prophylactic lateral node dissection was also performed for two patients.

The remaining eight patients (20.0\%) had coexisting PTC lesions in the thyroid, which were diagnosed on preoperative ultrasound and cytology. One patient showed clinical node metastasis of PTC in the lateral compartment. Six of the eight patients underwent a total thyroidectomy, and a lobectomy with isthmectomy was performed for the remaining two patients. All eight patients underwent central node dissection, and one N1b patient also underwent bilateral lateral node dissection.

\section{Pathological examination for NIFTP patients}

All of the NIFTP patients were diagnosed pathologically, and none of these patients showed lymph node metastasis of NIFTP. Six patients had pathological node metastasis of coexisting PTC. The Ki-67 labeling index (LI) for the NIFTP lesion was investigated in 36 cases. The LIs in hot spots of 35 cases were $<5 \%$, and that of the remaining patient was $5 \%-10 \%$.

\section{Postoperative follow-up}

A patient who had coexisting PTC with clinical node metastasis underwent radioactive iodine (RAI) ablation $(30 \mathrm{mCi})$ using recombinant human thyroid-stimulating hormone (TSH) after surgery, with no finding of suspicious metastatic lesions. We performed the postoperative follow-up mainly with blood examinations and ultrasound once or twice per year. The postoperative followup periods ranged from 7 to 150 months (median 80 months). To date, PTC recurrence occurred in one patient in the lateral lymph node and in one patient in subcutaneous tissue (suspected of needle tract implantation). These recurrences were successfully treated with redo surgery. None of the patients in our series showed a recurrence of NIFTP.

\section{Discussion}

To date, several reports have been published regarding the ultrasound findings of NIFTP in comparisons with other diseases such as FVPTC. With integrated evaluations, it was shown that NIFTP was less likely to be diagnosed as malignant on ultrasound based on the Korean Thyroid Imaging Reporting and Data System and on the Thyroid Imaging Reporting \& Data System (10, $13,14)$. With individualized evaluations of ultrasound findings, NIFTPs were less likely to show any types of calcification and speculated/microlobulated margin [11]. Brandler et al. [10] compared ultrasound findings of NIFTP with those of classic PTC and follicular adenoma, and they showed that compared to the class PTC cases, NIFTP was more likely to show a smooth border and isoechoic echogenicity and to lack calcifications, whereas no significant difference in ultrasound findings between their NIFTP and follicular adenoma cases were detected. These findings suggested that NIFTP is more likely to show benign findings on ultrasound than classic PTC and FVPTC, although NIFTP shows ultrasound findings that are similar to those of follicular adenoma.

In this study, we investigated the differences in ultrasound findings between NIFTP and FVPTC and between NIFTP and encap-PTC. Our analyses revealed that based on the diagnostic criteria issued by the JSUM [17], the detection rates of benign findings of NIFTP were high as follows: regular shape (62.5\%), a well-defined boundary zone $(82.5 \%)$, isoechoic echoes $(75.0 \%)$, homogeneous internal echoes $(85.0 \%)$, and lack of punctate microcalcification (95.0\%). We compared the ultrasound findings of NIFTP with those of FVPTC and encap-PTC, and our findings demonstrated that the incidences of benign findings in the JSUM criteria in our NIFTP group were significantly higher than those in the FVPTC and encapPTC groups (Fig. 1). In addition, as shown in Table 1, the number of benign findings based on the JSUM criteria in the NIFTP group was significantly higher than those in the FVPTC and encap-PTC groups. These findings suggest that the JSUM diagnostic criteria include many useful ultrasound findings to diagnose whether a nodule is malignant or benign. 
We next compared the ultrasound findings of NIFTP, FVPTC, and encap-PTC based on our USC classification system at Kuma Hospital [19], and we observed that $45 \%$ and $55 \%$ of the NIFTP cases were diagnosed as benign and borderline, respectively, and no cases were classified as malignant ( $\geq$ USC3.5). This is in sharp contrast to the ultrasound findings in the FVPTC and encapPTC cases: $54.8 \%$ and $53.2 \%$ were diagnosed as malignancy, respectively. Therefore, our USC classification system is also useful to discriminate nodules suspected of NIFTP, and based on this system, NIFTP is very unlikely to be diagnosed as a malignant tumor on ultrasound.

In our series, although a certain number of NIFTPs showed features that are indicative of malignancy based on the JSUM diagnostic criteria (i.e., irregular shape, jagged boundary, and irregular or no marginal hypoechoic zones), none of the NIFTPs were diagnosed as malignant based on the USC classification. This might be because of the difference in evaluation methods of nodules between the JSUM and USC systems. When the JSUM diagnostic criteria are used, various findings on ultrasound are individually checked and classified into two categories to diagnose whether each nodule is benign or malignant. In the USC system, in contrast, although there are some prominent points of evaluation (e.g., cystic or solid nodule, regular or irregular border, and extrathyroid extension), each nodule is evaluated and diagnosed more comprehensively than is done with the JSUM criteria. Sonograghers at Kuma Hospital grade the various ultrasound findings (e.g., weak, moderate, and strong) rather than classifying them into two categories, indicating that diagnoses based on the USC system depend more on the sonographers' experience level compared to the JSUM criteria, and constant precision management is required. These fundamental differences between the JSUM criteria and the USC system could explain, at least in part, the discrepancy in ultrasound diagnoses based on these two systems.

On cytology, in contrast, the rate of NIFTPs that were suspected of being or diagnosed as malignancy (Be-V or -VI) was $86.9 \%$. Although this rate is even lower than that of encap-PTC, it is still very high, indicating that most NIFTPs were suspected of being or diagnosed as malignant on cytology. Our results are similar to those of other studies in Japan [4, 5], whereas studies from Western countries demonstrated lower rates of $\mathrm{Be}-\mathrm{V}$ or -VI, i.e., from $23.7 \%$ to $45.9 \%$ [9]. The reasons for this discrepancy remain unknown. However, since NIFTP has a typical nuclear finding of PTC in at least some of the tumor cells, it is possible that NIFTPs are cytologically suspected of being or diagnosed as PTC at a high rate.

Indeed, the diagnosis of NIFTP based on ultrasound is still difficult because there are no ultrasound findings that are typical of NIFTP. However, as shown in Table 2, NIFTP has significantly more benign findings based on the JSUM criteria compared to FVPTC and encap-PTC, and none of the present NIFTPs were suspected of being or diagnosed as PTC based on our USC classification system (Table 4). We could therefore suspect nodules with no or few malignant findings as being NIFTP on ultrasound examination. Since most of these cases are cytologically suspected of being or diagnosed as PTC, it might be difficult to avoid surgery if the nodule measures $>10 \mathrm{~mm}$. However, the ultrasound findings indicating the possibility of NIFTP could help avoid extensive surgery (such as total thyroidectomy and prophylactic lateral node dissection) even if the tumor size is large. Of course, in this patient group, FVPTC and encap-PTC should be included to some extent; however, the prognoses both of low-risk FVPTC and encap-PTC were reported to be excellent, indicating that extensive surgery for these patients is not needed and should even be avoided $[21,22]$.

In our series, none of the patients showed lymph node metastasis of NIFTP. In addition, all cases but one had low growth activity as indicated by the Ki-67 LI, which is similar to a previous study [4]. During the postoperative follow-up (median 80 months), none of these patients showed recurrence of NIFTP. Together, all of the present findings strongly suggest that NIFTP is a very indolent disease, and if nodules are diagnosed as NIFTP on pathological examination and no other malignancies coexist, any additional therapies such as completion total thyroidectomy and adjuvant RAI therapy should not be done, to avoid overtreatment.

A novel definition of NIFTP was published in 2018 [23]; its authors proposed the following additional criteria: (1) no true papillary structures, (2) the exclusion of fully developed nuclear features (corresponding to nuclear grade 3) of PTC, and (3) the exclusion of mutations that are characteristic of conventional PTC or highrisk cancer, i.e., BRAFV600E, RET/PTC, and TERT. Based on these criteria, we could more strictly select pure NIFTPs before surgery and avoid overtreatment, including the extent of the thyroidectomy in the initial surgery.

This study has some limitations. It was a retrospective analysis, but more importantly, we enrolled NIFTP patients in the series of patients who were pathologically diagnosed as having FVPTC, which could be one of the reasons for the high rate of NIFTP cytologically diagnosed as or suspected of being PTC. If the cases of NIFTP patients were collected prospectively, the ultrasound and cytological findings would vary a good deal. Another limitation is the observer variation among 
sonographers. This study was based on the retrospective findings of one sonographer, but if other sonographers evaluate the same findings, their conclusions could be discrepant for a portion of the cases.

In summary, NIFTPs are rarely diagnosed as a malignancy on ultrasound, but they are frequently suspected of being or diagnosed as PTC on cytology, indicating that if the tumor size exceeds $10 \mathrm{~mm}$, it might be difficult to avoid surgical treatment. However, since NIFTPs can be diagnosed on ultrasound to some extent, we could avoid extensive surgery for such nodules by carefully evaluating the ultrasound findings. To avoid overtreatment, additional therapies such as completion total thyroidectomy and RAI therapy for NIFTP should not be performed.

\section{References}

1. Nikiforov YE, Seethala RR, Tallini G, Baloch ZW, Basolo F, et al. (2016) Nomenclature revision for encapsulated follicular variant of papillary thyroid carcinoma: a paradigm shift to reduce overtreatment of indolent tumors. JAMA Oncol 2: 1023-1029.

2. Lloyd RV, Osamura RY, Kroppel G, Rosai J (2017) WHO classification of tumours of endocrine organs (4th edition). IARC: Lyon, France.

3. Bychkov A, Hirokawa M, Jung CK, Liu Z, Zhu Y, et al. (2017) Low rate of NIFTP in Asian practice. Thyroid 27: 983-984.

4. Hirokawa M, Higuchi M, Suzuki A, Hayashi T, Kuma S, et al. (2017) Noninvasive follicular thyroid neoplasm with papillary-like nuclear features: a single-institutional experience. Endocr J 64: 1149-1155.

5. Koshikawa T, Fujita N, Ueda N, Ota Y, Sasaki E, et al. (2019) Important cytological findings for distinction between follicular variant and conventional papillary thyroid carcinoma, including noninvasive follicular thyroid tumors with papillary-like nuclear features. Endocr $J 66$ : 475-483.

6. Higuchi M, Hirokawa M, Kanematu R, Tanaka A, Suzuki A, et al. (2018) Impact of the modification of the diagnostic criteria in the 2017 Bethesda System for reporting thyroid cytopathology: a report of a single institution in Japan. Endocr J 65: 1193-1198.

7. Thompson LD (2016) Ninety-four cases of encapsulated follicular variant of papillary thyroid carcinoma: a name change to noninvasive follicular thyroid neoplasm with papillary-like nuclear features would help prevent overtreatment. Mod Pathol 29: 698-707.

8. Liu Z, Bychkov A, Jung CK, Hirokawa M, Sui S, et al. (2019) Intrerobserver and intraobserver variation in the morphological evaluation of noninvasive follicular neoplasm with papillary-like nuclear features in Asian practice. Pathology Int 69: 202-210.

9. Yan L, Sethi S, Park JW (2019) Cytologic and clinical features of NIFTP: can we diagnose based on preoperative fine-needle aspiration. Diagn Cytopathol 47: 1259-1266.

10. Brandler TC, Zhou F, Liu CZ, Cho M, Lau RP, et al. (2017) Can noninvasive follicular thyroid neoplasm with papillary-like nuclear features be distinguished from classic papillary thyroid carcinoma and follicular adenomas by fine-needle aspiration? Cancer Cytopathol 125: 378-
388.

11. Hahn SY, Shin JH, Lim HK, Jung SL, Oh YL, et al. (2017) Preoperative differentiation between noninvasive follicular thyroid neoplasm with papillary-like nuclear features (NIFTP) and non-NIFTP. Clin Endocrinol (Oxf) 86: 444-450.

12. Lee HS, Lee JW, Park JH, Kim WS, Han HS, et al. (2019) Comprehensive analysis for diagnosis of preoperative non-invasive follicular thyroid neoplasms with papillarylike nuclear features. PLoS One 14: e0218046.

13. Rosario PW, da Silva AL, Nunes MB, Borges MAR (2018) Risk of malignancy in thyroid nodules using the American College of Radiology Thyroid Imaging Reporting and Data System in the NIFTP era. Horm Metab Res 50: 735-737.

14. Bousier L, Clerc Urmes I, Garon J, Klein M, Demarquet L (2020) Ultrasound and cytological characteristics of noninvasive follicular thyroid neoplasm with papillary-like nuclear features compared to papillary carcinomas. Ann Endocrionl (Paris) 81: 28-33.

15. Rana C, Manjunath S, Ramakant P, Singh K, Babu S, et al. (2020) Noninvasive follicular neoplasm with papillary like nuclear features: a comprehensive analysis with a diagnostic algorithm. Diagn Cytopathol 48: 330-341.

16. Lam CA, McGettigan MJ, Thompson ZJ, Khazai L, Chung CH, et al. (2019) Ultrasound characterization for thyroid nodules with indeterminate cytology: interobserver agreement and impact of combining patternbased and scoring-based classifications in risk stratification. Endocrine 66: 278-287.

17. Kitaoka M, Miyamoto $\mathrm{Y}$, Fukunari N, Omoto K, Kameyama K, et al. (2011) Ultrasound diagnostic criteria for thyroid nodule. Jpn J Med Ultrasonics 38: 669-670.

18. Asteria C, Giovanardi A, Pizzocaro A, Cozzaglio L, Morabito A, et al. (2008) US-elastography in the differential diagnosis of benign and malignant thyroid nodules. Thyroid 18: 523-531.

19. Yokozawa T, Fukata S, Kuma K, Matsuzuka F, Kobayashi A, et al. (1996) Thyroid cancer detected by ultrasoundguided fine-needle aspiration biopsy. World J Surg 20: 848-853.

20. Pustaszeri M, Rossi ED, Auger M, Baloch X, Bishop J, et al. (2016) The Bethesda System for reporting thyroid cytopathology: proposed modifications and updates for the 
second edition from an international panel. Acta Cytol 60: 399-405.

21. Ito Y, Hirokawa M, Uruno T, Kihara M, Higashiyama T, et al. (2008) Biological behavior and prognosis of encapsulated papillary carcinoma of the thyroid: experience of a Japanese hospital for thyroid care. World J Surg 32: 1789 1794.

22. Koh J, Kim EK, Kim JY, Kwak JY, Yoon JH, et al. (2016) Comparison of ultrasound, pathological and prognostic characteristics of the follicular variant of papillary thyroid cancer according to fine-needle aspiration cytology. Ultrasound Med Biol 42: 2864-2872.

23. Alves VAF, Kakudo K, LiVolsi V, Lloyd RV, Nikiforov YE, et al. (2018) Noninvasive follicular thyroid neoplasm with papillary-like nuclear features (NIFTP): achieving better agreement by refining diagnostic criteria. Clinics (Sao Paulo) 73: e576. 